Rapid Reviews COVID-19

\title{
Review 2: "Evaluating aerosol and splatter during orthodontic debonding: implications for the COVID-19 pandemic"
}

Filippo Graziani ${ }^{1}$, Rossana Izzetti $^{\mathbf{2}}$

${ }^{1}$ University of Pisa School of Medicine and Surgery: Universita degli Studi di Pisa, Department of Surgical, Medical and Molecular Pathology and Critical Care, Medicine, Italy,

${ }^{2}$ Department of Translational Research on New Technologies in Medicine and Surgery, Università di Pisa

Published on: Oct 08, 2020

DOI: $10.1162 / 2$ e3983f5.aa92e751

License: Creative Commons Attribution 4.0 International License(CC-BY 4.0). 


\section{$\underline{\text { RR:C19 Evidence Scale rating by reviewer: }}$}

- Reliable. The main study claims are generally justified by its methods and data. The results and conclusions are likely to be similar to the hypothetical ideal study. There are some minor caveats or limitations, but they would/do not change the major claims of the study. The study provides sufficient strength of evidence on its own that its main claims should be considered actionable, with some room for future revision.

\footnotetext{
$* * * * * * * * * * * * * * * * * * * * * * * * * * * * * * * * * * * * * * * *$
}

\section{Review:}

We read with great interest the article by Llandro et al., ${ }^{1}$ which addresses a timely issue, and aims at assessing the impact of debonding procedures in orthodontics on aerosol production.

\section{Why is the study important?}

The study provides further insight into aerosol production in dental practice. Indeed, the recent diffusion of SARS-CoV-2 and the high viability of the virus through aerosol particles have led to an increasing interest towards aerosol generating procedures, which should be limited when possible. ${ }^{2,3}$ Interestingly, the issue of aerosol production in orthodontics is addressed and appears relevant in the current scenario being orthodontic procedures extremely diffused worldwide especially among younger patients, which could potentially be asymptomatic. ${ }^{4}$

\section{Lessons to be learned}

Orthodontic procedures do not seem related to a massive aerosol production. Overall, it was reported that aerosol and spatter deposition was detected only in the proximity of the dental chair, suggesting a low risk for aerosol generation following orthodontic debonding. However, cautious practice of dental procedures should be further reinforced.

\section{Potential issues with the study}

The study was performed by using a slow speed handpiece with dental suction. It would be of interest to retrieve data on aerosol production in absence of containment measures, in order to establish a benchmark and to assess whether the procedures suggested are effective to limit aerosol production. Moreover, phantom studies have 
important limitations due to the simulation of the salivary flow and the low specificity on aerosol evaluation versus spatter. Overall, the study design appears more suitable in the assessment of larger spatter rather than the evaluation of micro-aerosol, which is of utmost importance in the current scenario as the presence of the SARS-CoV-2 has been reported in submillimeter aerosol particles. ${ }^{5}$

\section{Further studies suggestion}

Being the issue of aerosol extremely relevant, we encourage the performance of in-vivo assessment of aerosol production, possibly integrated by the characterization of the aerosol particles produced in terms of size. Such an evaluation may be performed with the support of air samplers or particle counting instruments, as suggested by the authors. Finally, the performance of microbiological sampling may complete the assessment of the impact of aerosol generating procedures on air quality.

\section{Conclusions}

We are grateful to the authors for raising the issue of aerosol production following debonding procedures in orthodontics. Assessing whether there is a risk of increased production of micro-aerosol may contribute to better delineating the impact of aerosol production in dentistry, and to give insights into the actual risk of cross-infection related to the dental procedures.

\section{Bibliography}

1. Llandro H, Allison JR, Currie CC, Edwards DC, Bowes C, Durham J, Jakubovics N, Rostami N, Holliday R. Evaluating aerosol and splatter during orthodontic debonding: implications for the COVID-19 pandemic. 2020

2. Chen J. Pathogenicity and transmissibility of 2019-nCoV-A quick overview and comparison with other emerging viruses. Microbes Infect. 2020;22(2):69-71. doi:10.1016/j.micinf.2020.01.004.

3. Izzetti R, Nisi M, Gabriele M, Graziani F. COVID-19 Transmission in Dental Practice: Brief Review of Preventive Measures in Italy. J Dent Res. 2020 Aug;99(9):1030-1038. doi: 10.1177/0022034520920580. Epub 2020 Apr 17. PMID: 32302257. 
4. Rothe C, Schunk M, Sothmann P, Bretzel G, Froeschl G, Wallrauch C, et al. Transmission of 2019-nCoV Infection from an Asymptomatic Contact in Germany. N Engl J Med. 2020;382(10):970-971. doi:10.1056/NEJMc2001468

5. Liu Y, Ning Z, Chen Y, Guo M, Liu Y, Gali NK, et al. Aerodynamic characteristics and RNA concentration of SARS-CoV-2 aerosol in Wuhan Hospitals during COVID-19 outbreak. Nature 2020. doi: 10.1101/2020.03.08.982637. 